\title{
A Sharper Global Error Bound for the Generalized Nonlinear Complementarity Problem over a Polyhedral Cone
}

\author{
Hongchun Sun ${ }^{1}$ and Yiju Wang ${ }^{2}$ \\ ${ }^{1}$ School of Science, Linyi University, Linyi, Shandong 276005, China \\ ${ }^{2}$ School of Management Science, Qufu Normal University, Rizhao, Shandong 276800, China \\ Correspondence should be addressed to Yiju Wang; wyiju@hotmail.com
}

Received 12 July 2013; Revised 17 September 2013; Accepted 30 September 2013

Academic Editor: Abdellah Bnouhachem

Copyright (c) $2013 \mathrm{H}$. Sun and Y. Wang. This is an open access article distributed under the Creative Commons Attribution License, which permits unrestricted use, distribution, and reproduction in any medium, provided the original work is properly cited.

We revisit the global error bound for the generalized nonlinear complementarity problem over a polyhedral cone (GNCP). By establishing a new equivalent formulation of the GNCP, we establish a sharper global error bound for the GNCP under weaker conditions, which improves the existing error bound estimation for the problem.

\section{Introduction}

Let $\mathscr{K}=\left\{v \in R^{m} \mid A v \geq 0, B v=0\right\}$ be a polyhedral cone in $R^{m}$ for matrices $A \in R^{s \times m}, B \in R^{t \times m}$, and let $\mathscr{K}^{\circ}$ be its dual cone; that is,

$$
\mathscr{K}^{\circ}=\left\{u \in R^{m} \mid u=A^{\top} \lambda_{1}+B^{\top} \lambda_{2}, \lambda_{1} \in R_{+}^{s}, \lambda_{2} \in R^{t}\right\} .
$$

For continuous mappings $F, G: R^{n} \rightarrow R^{m}$, the generalized nonlinear complementarity problem, abbreviated as GNCP, is to find vector $x^{*} \in R^{n}$ such that

$$
F\left(x^{*}\right) \in \mathscr{K}, \quad G\left(x^{*}\right) \in \mathscr{K}^{\circ}, \quad F\left(x^{*}\right)^{\top} G\left(x^{*}\right)=0 .
$$

Throughout this paper, the solution set of the GNCP, denoted by $X^{*}$, is assumed to be nonempty.

The GNCP is a direct generalization of the classical nonlinear complementarity problem and a special case of the general variational inequalities problem [1]. The GNCP was deeply discussed [2-5] after the work in [6]. The GNCP plays a significant role in economics, operation research, nonlinear analysis, and so forth (see $[7,8])$. For example, the classical Walrasian law of competitive equilibria of exchange economies can be formulated as a generalized nonlinear complementarity problem in the price and excess demand variables (see [8]).
For the GNCP, the solution existence and the numerical solution methods for the GNCP were discussed $[2,3,6]$. As an important tool for a mathematical problem, the global error bound estimation for GNCP with the mapping being $\gamma$-strongly monotone and Hölder continuous was discussed in [5], and a global error bound for the GNCP for the linear and monotonic case was established in [4].

In this paper, we will establish a global error bound for the problem (2) without the Hölder continuity of the underlying mapping. To this end, we first develop some new equivalent reformulations of the GNCP under weaker conditions and then establish a sharper global error bound for the GNCP in terms of some easier computed residual functions. The results obtained in this paper can be taken as an improvement of the existing results for GNCP and variational inequalities problem $[4,5,9-11]$.

To end this section, we give some notations used in this paper. Vectors considered in this paper are taken in the Euclidean space $R^{n}$ equipped with the usual inner product, and the Euclidean 2-norm and 1-norm of vector in $R^{n}$ are, respectively, denoted by $\|\cdot\|$ and $\|\cdot\|_{1}$. We use $R_{+}^{n}$ to denote the nonnegative orthant in $R^{n}$ and use $x_{+}$and $x_{-}$to denote the vectors composed by elements $\left(x_{+}\right)_{i}:=\max \left\{x_{i}, 0\right\},\left(x_{-}\right)_{i}:=$ $\max \left\{-x_{i}, 0\right\}, 1 \leq i \leq n$, respectively. For simplicity, we use $(x ; y)$ to denote vector $\left(x^{\top}, y^{\top}\right)^{\top}$, use $I$ to denote the identity matrix with appropriate dimension, use $x \geq 0$ to denote 
a nonnegative vector $x \in R^{n}$, and use $\operatorname{dist}\left(x, X^{*}\right)$ to denote the distance from point $x$ to the solution set $X^{*}$.

\section{Global Error Bound for the GNCP}

First, we give some concepts used in the subsequent.

Definition 1. The mapping $F: R^{n} \rightarrow R^{m}$ is said to be

(i) monotone with respect to $G: R^{n} \rightarrow R^{m}$ if

$$
\langle F(x)-F(y), G(x)-G(y)\rangle \geq 0, \quad \forall x, y \in R^{n} ;
$$

(ii) $\gamma$-strongly $G$-monotone with respect to $G: R^{n} \rightarrow R^{m}$ if there are constants $c_{1}>0, \gamma>0$ such that

$$
\begin{array}{r}
\langle F(x)-F(y), G(x)-G(y)\rangle \geq c_{1}\|G(x)-G(y)\|^{1+\gamma}, \\
\forall x, y \in R^{n} .
\end{array}
$$

Remark 2. Based on this definition, $\gamma$-strongly $G$-monotone implies monotonicity, and if $F(x)=M x+p, G(x)=N x+q$ with $M, N \in R^{m \times n}, p, q \in R^{m}$, then the above Definition 1(i) is equivalent to that the matrix $M^{\top} N$ is positive semidefinite.

Now, we give some assumptions for our analysis based on Definition 1.

Assumption 3. For mappings $F, G$ and matrix $A$ involved in the GNCP, we assume that

(A1) mapping $F$ is monotone with respect to mapping $G$;

(A2) matrix $A^{\top}$ has full-column rank.

Remark 4. Under (A2) in the assumption, matrix $A^{\top}$ has left inverse $\left(A A^{\top}\right)^{-1} A$, that is, its pseudoinverse of $A^{\top}$. Certainly, the assumption on matrix $A^{\top}$ is weaker than that on matrix $\left(A^{\top}, B^{\top}\right)$ which has full-column rank [4]. In addition, when the mappings $F, G$ are both linear, then Assumption 3(A1) coincides with Assumption (A1) in [4].

In the following, we will establish a new equivalent reformulation to the GNCP. First, we give the following conclusion established in [2].

Theorem 5. A point $x^{*} \in R^{n}$ is a solution of the GNCP if and only if there exist $\lambda_{1}^{*} \in R^{s}, \lambda_{2}^{*} \in R^{t}$, such that

$$
\begin{gathered}
A F\left(x^{*}\right) \geq 0, \\
B F\left(x^{*}\right)=0, \\
\lambda_{1}^{*} \geq 0, \\
\left(F\left(x^{*}\right)\right)^{\top} G\left(x^{*}\right)=0, \\
G\left(x^{*}\right)=A^{\top} \lambda_{1}^{*}+B^{\top} \lambda_{2}^{*} .
\end{gathered}
$$

From Theorem 5, under Assumption 3(A2), we can transform the system into a new system in which neither $\lambda_{1}$ nor $\lambda_{2}$ is involved. To this end, we need the following conclusion [12].
Lemma 6. If the linear system $H y=b$ is consistent, then $y=$ $\mathrm{H}^{+} b$ is the solution with the minimum 2-norm, where $\mathrm{H}^{+}$is the pesudo-inverse of $H$.

Lemma 7. Suppose that Assumption 3(A2) holds. Then, for any $x \in R^{n}$, the following statements are equivalent.

(1) There exist $\lambda_{1} \in R_{+}^{s}, \lambda_{2} \in R^{t}$ such that $G(x)=A^{\top} \lambda_{1}+$ $B^{\top} \lambda_{2}$.

(2) Consider

$$
\begin{gathered}
\left\{-A_{L}^{-1} B^{\top}\left[\left(A^{\top} A_{L}^{-1}-I\right) B^{\top}\right]^{+}\left[A^{\top} A_{L}^{-1}-I\right]+A_{L}^{-1}\right\} G(x) \geq 0, \\
\left\{A^{\top}\left\{-A_{L}^{-1} B^{\top}\left[\left(A^{\top} A_{L}^{-1}-I\right) B^{\top}\right]^{+}\left[A^{\top} A_{L}^{-1}-I\right]+A_{L}^{-1}\right\}\right. \\
\left.+B^{\top}\left[\left(A^{\top} A_{L}^{-1}-I\right) B^{\top}\right]^{+}\left[A^{\top} A_{L}^{-1}-I\right]-I\right\} G(x)=0,
\end{gathered}
$$

where $A_{L}^{-1}=\left(A A^{\top}\right)^{-1} A$.

Proof. The proof follows that of Lemma 2.1 in [4], and for completeness, we include it.

$$
\text { Set }
$$

$$
\begin{aligned}
& X_{1}:=\left\{x \in R^{n} \mid G(x)=A^{\top} \lambda_{1}+B^{\top} \lambda_{2}\right. \\
& \text { for some } \left.\lambda_{1} \in R_{+}^{s}, \lambda_{2} \in R^{t}\right\}, \\
& X_{2}:=\left\{x \in R^{n} \mid\left\{-A_{L}^{-1} B^{\top}\left[\left(A^{\top} A_{L}^{-1}-I\right) B^{\top}\right]^{+}\right.\right. \\
& \left.\times\left[A^{\top} A_{L}^{-1}-I\right]+A_{L}^{-1}\right\} G(x) \geq 0, \\
& \left\{A ^ { \top } \left\{-A_{L}^{-1} B^{\top}\left[\left(A^{\top} A_{L}^{-1}-I\right) B^{\top}\right]^{+}\right.\right. \\
& \left.\times\left[A^{\top} A_{L}^{-1}-I\right]+A_{L}^{-1}\right\} \\
& +B^{\top}\left[\left(A^{\top} A_{L}^{-1}-I\right) B^{\top}\right]^{+} \\
& \left.\left.\times\left[A^{\top} A_{L}^{-1}-I\right]-I\right\} G(x)=0\right\} .
\end{aligned}
$$

Now, we show that these two sets are equal.

First, for any $x \in X_{1}$, there exist $\lambda_{1} \in R_{+}^{s}, \lambda_{2} \in R^{t}$ such that

$$
G(x)=A^{\top} \lambda_{1}+B^{\top} \lambda_{2}
$$

Premultiplying (8) by $A_{L}^{-1}:=\left(A A^{\top}\right)^{-1} A$ gives

$$
A_{L}^{-1} G(x)=\lambda_{1}+A_{L}^{-1} B^{\top} \lambda_{2}
$$

Combining this with (8) yields that

$$
\begin{aligned}
G(x) & =A^{\top}\left(A_{L}^{-1} G(x)-A_{L}^{-1} B^{\top} \lambda_{2}\right)+B^{\top} \lambda_{2} \\
& =A^{\top} A_{L}^{-1} G(x)-\left[A^{\top} A_{L}^{-1} B^{\top}-B^{\top}\right] \lambda_{2} ;
\end{aligned}
$$


that is,

$$
\left[A^{\top} A_{L}^{-1} B^{\top}-B^{\top}\right] \lambda_{2}=\left[A^{\top} A_{L}^{-1}-I\right] G(x) .
$$

Recalling Lemma 6, we further have

$$
\lambda_{2}=\left[\left(A^{\top} A_{L}^{-1}-I\right) B^{\top}\right]^{+}\left[A^{\top} A_{L}^{-1}-I\right] G(x) .
$$

Combining this with (9) yields that

$$
\begin{aligned}
\lambda_{1}= & \left\{-A_{L}^{-1} B^{\top}\left[\left(A^{\top} A_{L}^{-1}-I\right) B^{\top}\right]^{+}\left[A^{\top} A_{L}^{-1}-I\right]+A_{L}^{-1}\right\} \\
& \times G(x) .
\end{aligned}
$$

Using (8), (12), and (13), we have

$$
\begin{aligned}
& \left\{A^{\top}\left\{-A_{L}^{-1} B^{\top}\left[\left(A^{\top} A_{L}^{-1}-I\right) B^{\top}\right]^{+}\left[A^{\top} A_{L}^{-1}-I\right]+A_{L}^{-1}\right\}\right. \\
& \left.+B^{\top}\left[\left(A^{\top} A_{L}^{-1}-I\right) B^{\top}\right]^{+}\left[A^{\top} A_{L}^{-1}-I\right]-I\right\} G(x)=0 .
\end{aligned}
$$

From the fact that $\lambda_{1} \geq 0$, by (13), one has

$$
\begin{aligned}
& \left\{-A_{L}^{-1} B^{\top}\left[\left(A^{\top} A_{L}^{-1}-I\right) B^{\top}\right]^{+}\left[A^{\top} A_{L}^{-1}-I\right]+A_{L}^{-1}\right\} G(x) \\
& \quad \geq 0 .
\end{aligned}
$$

Combining this with (14) leads to that $x \in X_{2}$. This shows that $X_{1} \subseteq X_{2}$.

Second, for any $x \in X_{2}$, let

$$
\begin{aligned}
& \lambda_{1}=\left\{-A_{L}^{-1} B^{\top}\left[\left(A^{\top} A_{L}^{-1}-I\right) B^{\top}\right]^{+}\left[A^{\top} A_{L}^{-1}-I\right]+A_{L}^{-1}\right\} \\
& \times G(x), \\
& \lambda_{2}=\left\{\left[\left(A^{\top} A_{L}^{-1}-I\right) B^{\top}\right]^{+}\left[A^{\top} A_{L}^{-1}-I\right]\right\} G(x) .
\end{aligned}
$$

Then, $\lambda_{1} \in R_{+}^{s}, \lambda_{2} \in R^{t}$. From (14), one has

$$
\begin{aligned}
G(x)= & A^{\top}\left\{-A_{L}^{-1} B^{\top}\left[\left(A^{\top} A_{L}^{-1}-I\right) B^{\top}\right]^{+}\left[A^{\top} A_{L}^{-1}-I\right]+A_{L}^{-1}\right\} \\
& \times G(x) \\
& +B^{\top}\left\{\left[\left(A^{\top} A_{L}^{-1}-I\right) B^{\top}\right]^{+}\left[A^{\top} A_{L}^{-1}-I\right]\right\} G(x) \\
= & A^{\top} \lambda_{1}+B^{\top} \lambda_{2} ;
\end{aligned}
$$

that is, $x \in X_{1}$. Hence, $X_{2} \subseteq X_{1}$, and the desired result follows.
Combining this conclusion with Theorem 5, we can establish the following equivalent formulation of the GNCP:

$$
A F(x) \geq 0,
$$$$
B F(x)=0,
$$

$$
(F(x))^{\top} G(x)=0,
$$$$
U G(x) \geq 0,
$$$$
V G(x)=0 \text {, }
$$

where

$$
\begin{aligned}
& U=\left\{-A_{L}^{-1} B^{\top}\right. {\left.\left[\left(A^{\top} A_{L}^{-1}-I\right) B^{\top}\right]^{+}\left[A^{\top} A_{L}^{-1}-I\right]+A_{L}^{-1}\right\}, } \\
& V=\left\{A ^ { \top } \left\{-A_{L}^{-1} B^{\top}\left[\left(A^{\top} A_{L}^{-1}-I\right) B^{\top}\right]^{+}\right.\right. \\
&\left.\times\left[A^{\top} A_{L}^{-1}-I\right]+A_{L}^{-1}\right\} \\
&\left.+B^{\top}\left[\left(A^{\top} A_{L}^{-1}-I\right) B^{\top}\right]^{+}\left[A^{\top} A_{L}^{-1}-I\right]-I\right\} .
\end{aligned}
$$

For the ease of description, we denote $\mu=F(x), \nu=G(x)$. Thus, system (18) can be written as

$$
\begin{gathered}
A \mu \geq 0, \\
B \mu=0, \\
\mu^{\top} v=0, \\
U \nu \geq 0, \\
V \nu=0 .
\end{gathered}
$$

For system (20), one has

$$
\begin{aligned}
& \mu^{\top} \nu=\mu^{\top}\left\{A^{\top}\{\right.-A_{L}^{-1} B^{\top}\left[\left(A^{\top} A_{L}^{-1}-I\right) B^{\top}\right]^{+} \\
&\left.\times\left[A^{\top} A_{L}^{-1}-I\right]+A_{L}^{-1}\right\} \\
&+B^{\top} {\left.\left[\left(A^{\top} A_{L}^{-1}-I\right) B^{\top}\right]^{+}\left[A^{\top} A_{L}^{-1}-I\right]\right\} \nu } \\
&=[A \mu]^{\top}\{-A_{L}^{-1} B^{\top}\left[\left(A^{\top} A_{L}^{-1}-I\right) B^{\top}\right]^{+} \\
& \times {\left.\left[A^{\top} A_{L}^{-1}-I\right]+A_{L}^{-1}\right\} \nu } \\
&+[B \mu]^{\top}\left\{\left[\left(A^{\top} A_{L}^{-1}-I\right) B^{\top}\right]^{+}\left[A^{\top} A_{L}^{-1}-I\right]\right\} \nu \\
&=[A \mu]^{\top}[U \nu],
\end{aligned}
$$

where the first equality follows from the last equality in (20), and the last equality uses the second equality in (20). Thus, system (20) can be further written as

$$
\begin{gathered}
A \mu \geq 0, \quad B \mu=0, \\
(A \mu)^{\top}(U \nu)=0, \\
U \nu \geq 0, \quad V \nu=0 .
\end{gathered}
$$


Furthermore, for any $(\mu, \nu) \in R^{m} \times R^{m}$ with $A \mu \geq 0, U v \geq 0$, it holds from (21) that

$$
\mu^{\top} v \geq 0 .
$$

Now, consider the following optimization problem:

$$
\begin{array}{ll}
\min & f(\omega)=[(I, 0) \omega]^{\top}[(0, I) \omega] \\
\text { s.t. } & \omega \in \Omega,
\end{array}
$$

where $\omega=(\mu, \nu), \Omega=\left\{\omega \in R^{2 m} \mid A(I, 0) \omega \geq 0, B(I, 0) \omega=0\right.$, $U(0, I) \omega \geq 0, V(0, I) \omega=0\}$. Denote the solution set of (24) by $\Omega^{*}$.

Lemma 8. Under Assumption 3(A1), $f(\omega)$ is a convex function.

Proof. For any $\omega_{1}, \omega_{2} \in R^{2 m}, \tau \in[0,1]$, we have

$$
\begin{aligned}
f\left(\tau \omega_{1}+\right. & \left.(1-\tau) \omega_{2}\right)-\tau f\left(\omega_{1}\right)-(1-\tau) f\left(\omega_{2}\right) \\
= & {\left[(I, 0)\left(\tau \omega_{1}+(1-\tau) \omega_{2}\right)\right]^{\top} } \\
& \times\left[(0, I)\left(\tau \omega_{1}+(1-\tau) \omega_{2}\right)\right] \\
& -\tau\left[(I, 0) \omega_{1}\right]^{\top}\left[(0, I) \omega_{1}\right] \\
& -(1-\tau)\left[(I, 0) \omega_{2}\right]^{\top}\left[(0, I) \omega_{2}\right] \\
= & \tau^{2}\left[(I, 0) \omega_{1}\right]^{\top}\left[(0, I) \omega_{1}\right] \\
& +(1-\tau)^{2}\left[(I, 0) \omega_{2}\right]^{\top}\left[(0, I) \omega_{2}\right] \\
& +\tau(1-\tau)\left[(I, 0) \omega_{1}\right]^{\top}\left[(0, I) \omega_{2}\right] \\
& +\tau(1-\tau)\left[(I, 0) \omega_{2}\right]^{\top}\left[(0, I) \omega_{1}\right] \\
& -\tau\left[(I, 0) \omega_{1}\right]^{\top}\left[(0, I) \omega_{1}\right] \\
& -(1-\tau)\left[(I, 0) \omega_{2}\right]^{\top}\left[(0, I) \omega_{2}\right] \\
= & -\tau(1-\tau)\left[(I, 0) \omega_{1}\right]^{\top}\left[(0, I) \omega_{1}\right] \\
& -\tau(1-\tau)\left[(I, 0) \omega_{2}\right]^{\top}\left[(0, I) \omega_{2}\right] \\
& +\tau(1-\tau)\left[(I, 0) \omega_{1}\right]^{\top}\left[(0, I) \omega_{2}\right] \\
& +\tau(1-\tau)\left[(I, 0) \omega_{2}\right]^{\top}\left[(0, I) \omega_{1}\right] \\
= & -\tau(1-\tau)\left(\left[(I, 0) \omega_{1}\right]-\left[(I, 0) \omega_{2}\right]\right)^{\top} \\
& \times\left((0, I) \omega_{1}-(0, I) \omega_{2}\right) \leq 0, \\
& +\tau(0)
\end{aligned}
$$

where the first inequality uses Assumption 3(A1). The desired result follows.

Based on (20), combining (23) with Lemma 8, we can obtain the following conclusion.

Lemma 9. A point $\omega^{*}=\left(\mu^{*}, \nu^{*}\right) \in R^{2 m}$ is a solution of (20) if and only if $\omega^{*}$ is a global optimal solution with the objective vanishing of (24).
In the following, we give the error bound for a polyhedral cone from [13] and error bound for a convex optimization from [14] to reach our aims.

Lemma 10. For polyhedral cone $P=\left\{x \in R^{n} \mid D_{1} x=\right.$ $\left.d_{1}, B_{1} x \leq b_{1}\right\}$ with $D_{1} \in R^{l \times n}, B_{1} \in R^{m \times n}, d_{1} \in R^{l}$, and $b_{1} \in$ $R^{m}$, there exists a constant $c_{2}>0$ such that

$$
\begin{array}{r}
\operatorname{dist}(x, P) \leq c_{2}\left[\left\|D_{1} x-d_{1}\right\|+\left\|\left(B_{1} x-b_{1}\right)_{+}\right\|\right], \\
\forall x \in R^{n} .
\end{array}
$$

Lemma 11. Let $P$ be a convex polyhedron in $R^{n}$, and let $\theta$ be a convex quadratic function defined on $R^{n}$. Let $S$ be the nonempty set of globally optimal solutions of the programming:

$$
\begin{array}{ll}
\min & \theta(x) \\
\text { s.t. } & x \in P
\end{array}
$$

with $\theta_{\text {opt }}$ being the optimal value of $\theta$ on $S$. There exists a scalar $c_{3}>0$ such that

$$
\begin{aligned}
\operatorname{dist}(x, S) \leq \mathcal{c}_{3} \max \left\{\operatorname{dist}(x, P),\left|\left[\theta(x)-\theta_{o p t}\right]_{+}\right|,\right. & \\
\left.\left|\left[\theta(x)-\theta_{o p t}\right]_{+}\right|^{1 / 2}\right\} & , \\
& \forall x \in R^{n} .
\end{aligned}
$$

Before proceeding, we present the following definition introduced in [15].

Definition 12. The mapping $G: R^{n} \rightarrow R^{m}$ is said to be strongly nonexpanding with a constant $\alpha>0$ if $\| G(x)-$ $G(y)\|\geq \alpha\| x-y \|$.

By Lemma $8, f(\omega)$ is a convex function and the feasible set $\Omega$ is a polyhedral. Combining this with Lemmas 10 and 11, we immediately obtain the following conclusion.

Theorem 13. Suppose that $F$ is $\gamma$-strongly G-monotone with positive constants $c_{1}, \gamma$, respectively, and $G$ is strongly nonexpanding with constant $\alpha>0$. Then, there exists constant $\rho_{1}>0$ such that

$$
\begin{aligned}
& \operatorname{dist}\left(x, X^{*}\right) \leq \rho_{1}\{\left\|[A F(x)]_{-}\right\|+\|B F(x)\| \\
&+\left\|[U G(x)]_{-}\right\|+\|V G(x)\| \\
&+\left|\left[F(x)^{\top} G(x)\right]_{+}\right| \\
&\left.+\left|\left[F(x)^{\top} G(x)\right]_{+}\right|^{1 / 2}\right\}^{2 /(1+\gamma)}, \\
& \forall x \in R^{n} .
\end{aligned}
$$


Proof. For any $x \in R^{n}$, let $\omega=(\mu, v)=(F(x), G(x)) \in R^{2 m}$. Then, there exists $\omega^{*}=\left(\mu^{*}, \nu^{*}\right)=\left(F\left(x^{*}\right), G\left(x^{*}\right)\right) \in \Omega^{*}$ such that $\operatorname{dist}\left(\omega, \Omega^{*}\right)=\left\|\omega-\omega^{*}\right\|$. A direct computation yields that

$$
\begin{aligned}
& \operatorname{dist}^{1+\gamma}\left(x, X^{*}\right) \\
& \leq\left\|x-x^{*}\right\|^{1+\gamma} \\
& \leq \frac{1}{\alpha^{1+\gamma}}\left\|G(x)-G\left(x^{*}\right)\right\|^{1+\gamma} \\
& \leq \frac{1}{c_{1} \alpha^{1+\gamma}}\left[\left(F(x)-F\left(x^{*}\right)\right)^{\top}\left(G(x)-G\left(x^{*}\right)\right)\right] \\
& \leq \frac{1}{c_{1} \alpha^{1+\gamma}}\left\|F(x)-F\left(x^{*}\right)\right\|\left\|G(x)-G\left(x^{*}\right)\right\| \\
& \leq \frac{1}{2 c_{1} \alpha^{1+\gamma}}\left\{\left\|F(x)-F\left(x^{*}\right)\right\|^{2}+\left\|G(x)-G\left(x^{*}\right)\right\|^{2}\right\} \\
& =\frac{1}{2 c_{1} \alpha^{1+\gamma}}\left\|\omega-\omega^{*}\right\|^{2} \\
& =\frac{1}{2 c_{1} \alpha^{1+\gamma}} \operatorname{dist}^{2}\left(\omega, \Omega^{*}\right) \\
& \leq \frac{1}{2 c_{1} \alpha^{1+\gamma}} c_{3}^{2} \\
& \times \max \left\{\operatorname{dist}(\omega, \Omega),\left|[f(\omega)]_{+}\right|,\left|[f(\omega)]_{+}\right|^{1 / 2}\right\}^{2} \\
& \leq \frac{1}{2 c_{1} \alpha^{1+\gamma}} c_{3}^{2} \\
& \times \max \left\{c _ { 2 } \left\{\left\|[A(I, 0) \omega]_{-}\right\|+\|B(I, 0) \omega\|\right.\right. \\
& \left.+\left\|[U(0, I) \omega]_{-}\right\|+\|V(0, I) \omega\|\right\}, \\
& \left.\left|[f(\omega)]_{+}\right|,\left|[f(\omega)]_{+}\right|^{1 / 2}\right\}^{2} \\
& \leq \frac{1}{2 c_{1} \alpha^{1+\gamma}} c_{3}^{2} \\
& \times \max \left\{c_{2}, 1\right\}^{2}\left\{\left\|[A \mu]_{-}\right\|+\|B \mu\|\right. \\
& +\left\|[U \nu]_{-}\right\|+\|V \nu\| \\
& \left.+\left|\left[\mu^{\top} \nu\right]_{+}\right|+\left|\left[\mu^{\top} \nu\right]_{+}\right|^{1 / 2}\right\}^{2},
\end{aligned}
$$

where the second inequality follows from Definition 12 with constant $\alpha>0$, the third inequality follows from Definition 1(ii) with constants $c_{1}>0, \gamma>0$, the fourth inequality follows from the Cauchy-Schwarz inequality, the fifth inequality follows from the fact that $(1 / 2)\left(a^{2}+b^{2}\right) \geq a b$, for all $a, b \in R$, the sixth inequality follows from Lemma 11 with constant $c_{3}>0$ and Lemma 9 , and the seventh inequality follows from Lemma 10 with constant $c_{2}>0$. By (30) and letting $\rho_{1}=\left\{\left(1 / 2 c_{1} \alpha^{1+\gamma}\right) c_{3}^{2} \max \left\{c_{2}, 1\right\}^{2}\right\}^{1 /(1+\gamma)}$, then the desired result follows.
Remark 14. It is clear that if $F$ is $\gamma$-strongly $G$-monotone and $G$ is strongly nonexpanding, then

$$
\begin{array}{r}
\langle F(x)-F(y), G(x)-G(y)\rangle \geq c_{1}\|G(x)-G(y)\|^{1+\gamma} \\
\geq c_{1} \alpha^{1+\gamma}\|x-y\|^{1+\gamma}, \\
\forall x, y \in R^{n} .
\end{array}
$$

Moreover, the conditions which both $F$ and $G$ are Hölder continuous (or both $F$ and $G$ are Lipschitz continuous) in Theorem 13 are removed. Thus, Theorem 13 is stronger than Theorem 2.5 in [5]. Furthermore, by Theorem 2.1 in [5], the GNCP can be reformulated as general variational inequalities problem, and the conditions in Theorem 13 are also weaker than those in Theorem 3.1 in [15], Theorem 3.1 in [11], Theorem 3.1 in [10], and Theorem 2 in [9], respectively.

On the other hand, the condition that $F$ is $\gamma$-strongly $G$ monotone and $G$ is strongly nonexpanding in Theorem 13 is extended compared with the condition that $F$ is strongly monotone with respect to $G$ (i.e., $\gamma=1$ ) in Theorems 3.4 and 3.6 in [15], and it is also extended than compared with the condition $F$ is strongly monotone with respect to $G$ (i.e., $\gamma=$ 1) in Theorem 3.1 in [11], and compared with the condition that $F(x)=x, G(x)$ is strongly monotone (i.e., $\gamma=1$ ) in Theorem 3.1 in [10].

Using the following Definition 15 developed from the complementarity conditions in (22), we can further detect the error bound of the GNCP.

Definition 15. A solution $x_{0}$ of the GNCP is said to be nondegenerate if it satisfies

$$
A F\left(x_{0}\right)+U G\left(x_{0}\right)>0 .
$$

Lemma 16. Suppose that Assumptions 3(A1) and 3(A2) hold, and the GNCP has a nondegenerate solution, say $x_{0}$. Then,

$$
\begin{aligned}
\Omega^{*}=\{\omega \in \Omega \mid & {[(I, 0) \omega]^{\top}\left[(0, I) \omega_{0}\right] } \\
+ & {\left.[(0, I) \omega]^{\top}\left[(I, 0) \omega_{0}\right]=0\right\}, }
\end{aligned}
$$

where $\omega_{0}=\left(\mu_{0}, v_{0}\right)=\left(F\left(x_{0}\right), G\left(x_{0}\right)\right)$.

Proof. Since

$$
\left[(I, 0) \omega_{0}\right]^{\top}\left[(0, I) \omega_{0}\right]=0,
$$

by Assumption 3(A1), for any $\omega \in \Omega$, we have

$$
\begin{aligned}
0 \leq & \left(\mu-\mu_{0}\right)^{\top}\left(\nu-v_{0}\right) \\
= & {\left[(I, 0) \omega-(I, 0) \omega_{0}\right]^{\top}\left[(0, I) \omega-(0, I) \omega_{0}\right] } \\
= & {[(I, 0) \omega]^{\top}[(0, I) \omega]-[(I, 0) \omega]^{\top}\left[(0, I) \omega_{0}\right] } \\
& -[(0, I) \omega]^{\top}\left[(I, 0) \omega_{0}\right] ;
\end{aligned}
$$

that is,

$$
\begin{gathered}
{[(I, 0) \omega]^{\top}\left[(0, I) \omega_{0}\right]+[(0, I) \omega]^{\top}\left[(I, 0) \omega_{0}\right]} \\
\leq[(I, 0) \omega]^{\top}[(0, I) \omega] .
\end{gathered}
$$


To prove the assertion, we only need to show that the solution set $\Omega^{*}$ is equal to the set

$$
\begin{aligned}
W:= & \left\{\omega \in \Omega \mid[(I, 0) \omega]^{\top}\left[(0, I) \omega_{0}\right]\right. \\
& \left.+[(0, I) \omega]^{\top}\left[(I, 0) \omega_{0}\right]=0\right\} .
\end{aligned}
$$

For any $\widetilde{\omega} \in \Omega^{*}$, combining Lemma 9 with (20) yields that

$$
[(I, 0) \widetilde{\omega}]^{\top}[(0, I) \widetilde{\omega}]=0 .
$$

Letting $\omega=\widetilde{\omega}$ in (36) yields that

$$
[(I, 0) \widetilde{\omega}]^{\top}\left[(0, I) \omega_{0}\right]+[(0, I) \widetilde{\omega}]^{\top}\left[(I, 0) \omega_{0}\right] \leq 0 .
$$

Since $\widetilde{\omega}, \omega_{0} \in \Omega$, using the similar technique to that of (21), we can obtain

$$
\begin{aligned}
{[(I, 0) \widetilde{\omega}]^{\top}\left[(0, I) \omega_{0}\right]+[(0, I) \widetilde{\omega}]^{\top}\left[(I, 0) \omega_{0}\right] } \\
\quad=\tilde{\mu}^{\top} v_{0}+\widetilde{v}^{\top} \mu_{0} \\
\quad=(A \widetilde{\mu})^{\top}\left(U v_{0}\right)+(U \widetilde{v})^{\top}\left(A \mu_{0}\right) \\
\quad \geq 0
\end{aligned}
$$

where $\widetilde{\omega}=(\widetilde{\mu}, \widetilde{\nu})$. Combining (39) with (40), we have $\Omega^{*} \subseteq$ $W$.

On the other hand, for any $\omega \in W$, one has

$$
[(I, 0) \omega]^{\top}\left[(0, I) \omega_{0}\right]+[(0, I) \omega]^{\top}\left[(I, 0) \omega_{0}\right]=0
$$

Since $\omega, \omega_{0} \in \Omega$, using the similar arguments to that of (21), one has

$$
\begin{aligned}
& {[(I, 0) \omega]^{\top}\left[(0, I) \omega_{0}\right]=[A \mu]^{\top}\left[U \nu_{0}\right]} \\
& {[(0, I) \omega]^{\top}\left[(I, 0) \omega_{0}\right]=[U \nu]^{\top}\left[A \mu_{0}\right]}
\end{aligned}
$$

Combining this with (41) yields that

$$
[A \mu]^{\top}\left[U \nu_{0}\right]+[U \nu]^{\top}\left[A \mu_{0}\right]=0 .
$$

From (32), we deduce that

$$
[A \mu]^{\top}[U \nu]=0
$$

Thus, using (21), one has

$$
\mu^{\top} v=0
$$

Hence, $\omega \in \Omega^{*}$.

Based on Lemma 16, we obtain the following conclusion.

Corollary 17. Suppose that the hypotheses of Lemma 16 hold. Then,

$$
\begin{aligned}
\Omega^{*}= & \left\{\omega \in \Omega \mid[(I, 0) \omega]^{\top}\left[(0, I) \omega_{0}\right]\right. \\
& \left.+[(0, I) \omega]^{\top}\left[(I, 0) \omega_{0}\right] \leq 0\right\}
\end{aligned}
$$

Theorem 18. Suppose that the hypotheses of Theorem 13 hold, and the GNCP has a nondegenerate solution. Then, there exists constant $\rho_{2}>0$ such that

$$
\begin{aligned}
& \operatorname{dist}\left(x, X^{*}\right) \\
& \leq \rho_{2}\left\{\left\|[A F(x)]_{-}\right\|+\|B F(x)\|+\left\|[U G(x)]_{-}\right\|\right. \\
& \left.\quad+\|V G(x)\|+\left|\left[F(x)^{\top} G(x)\right]_{+}\right|\right\}^{2 /(1+\gamma)}, \quad \forall x \in R^{n} .
\end{aligned}
$$

Proof. For any $x \in R^{n}$, let $\omega=(\mu, v)=(F(x), G(x)) \in R^{2 m}$. Then, there exists $\omega^{*}=\left(\mu^{*}, \nu^{*}\right)=\left(F\left(x^{*}\right), G\left(x^{*}\right)\right) \in \Omega^{*}$ such that $\operatorname{dist}\left(\omega, \Omega^{*}\right)=\left\|\omega-\omega^{*}\right\|$. Letting $x_{0}$ be a nondegenerate solution of GNCP and letting $\omega_{0}=\left(F\left(x_{0}\right), G\left(x_{0}\right)\right) \in \Omega^{*}$, then

$$
\begin{aligned}
& \operatorname{dist}^{1+\gamma}\left(x, X^{*}\right) \\
& \leq\left\|x-x^{*}\right\|^{1+\gamma} \\
& \leq \frac{1}{2 c_{1} \alpha^{1+\gamma}} \operatorname{dist}^{2}\left(\omega, \Omega^{*}\right) \\
& \leq \frac{1}{2 c_{1} \alpha^{1+\gamma}} c_{4}^{2} \\
& \times\left\{\left\|[A(I, 0) \omega]_{-}\right\|+\left\|[U(0, I) \omega]_{-}\right\|\right. \\
& +\|B(I, 0) \omega\|+\|V(0, I) \omega\| \\
& +\|\left\{[(I, 0) \omega]^{\top}\left[(0, I) \omega_{0}\right]\right. \\
& \left.\left.+[(0, I) \omega]^{\top}\left[(I, 0) \omega_{0}\right]\right\}_{+} \|\right\}^{2} \\
& \leq \frac{1}{2 c_{1} \alpha^{1+\gamma}} c_{4}^{2} \\
& \times\left\{\left\|[A(I, 0) \omega]_{-}\right\|+\left\|[U(0, I) \omega]_{-}\right\|\right. \\
& +\|B(I, 0) \omega\|+\|V(0, I) \omega\| \\
& \left.+\left\|\left\{[(I, 0) \omega]^{\top}[(0, I) \omega]\right\}_{+}\right\|\right\}^{2} \\
& =\frac{1}{2 c_{1} \alpha^{1+\gamma}} c_{4}^{2} \\
& \times\left\{\left\|[A \mu]_{-}\right\|+\left\|[U \nu]_{-}\right\|+\|B \mu\|\right. \\
& \left.+\|V \nu\|+\left\|\left\{\mu^{\top} \nu\right\}_{+}\right\|\right\}^{2},
\end{aligned}
$$

where the second equality uses the similar technique to that of (30), the third inequality follows from Corollary 17 and Lemma 10 with constant $c_{4}>0$, and the last inequality is based on (36). By (48) and letting $\rho_{2}=\left\{\left(1 / 2 c_{1} \alpha^{1+\gamma}\right) c_{4}^{2}\right\}^{1 /(1+\gamma)}$, the desired result follows.

In the following, we give an error bound of the Hölderian type [14].

Lemma 19. For $i=1,2, \ldots, m$, let $g_{i}(x)$ be a convex quadratic function. If the set $S:=\left\{x \in R^{n} \mid g_{1}(x) \leq 0\right.$, 
$\left.g_{2}(x) \leq 0, \ldots, g_{m}(x) \leq 0\right\}$ is nonempty, then there exist a positive integer $d \leq n+1$ (called the degree of singularity of the inequality system) and a positive scalar $c_{5}$ such that

$\operatorname{dist}(x, S) \leq c_{5} \max \left\{\left\|[g(x)]_{+}\right\|,\left\|[g(x)]_{+}\right\|^{1 / 2^{d}}\right\}, \quad \forall x \in R^{n}$,

where $[g(x)]_{+}=\left(\left[g_{1}(x)\right]_{+},\left[g_{2}(x)\right]_{+}, \ldots,\left[g_{m}(x)\right]_{+}\right)$. Furthermore, if $S$ contains an interior point, then $d=0$.

Based on (18) and (21), the GNCP can also be written as

$$
\begin{gathered}
A F(x) \geq 0, \\
B F(x)=0, \\
(F(x))^{\top} G(x) \leq 0, \\
U G(x) \geq 0, \\
V G(x)=0 .
\end{gathered}
$$

From Lemma 19, we can establish the following global error bound for GNCP.

Theorem 20. Suppose that the hypotheses of Theorem 13 hold, and there exists point $\widehat{x} \in R^{n}$ such that

$$
F(\widehat{x})^{\top} G(\widehat{x})<0
$$

Then, there exists constant $\rho_{3}>0$ such that

$$
\begin{aligned}
\operatorname{dist}\left(x, X^{*}\right) \leq \rho_{3}\{ & \left\|[A F(x)]_{-}\right\|+\|B F(x)\| \\
& +\left\|[U G(x)]_{-}\right\|+\|V G(x)\| \\
& \left.+\left|\left[F(x)^{\top} G(x)\right]_{+}\right|\right\}^{2 /(1+\gamma)}, \quad \forall x \in R^{n} .
\end{aligned}
$$

Proof. Let $S_{1}:=\left\{\omega \in R^{2 m} \mid f(\omega) \leq 0\right\}$, where $f(\omega)=[(I, 0) \omega]^{\top}[(0, I) \omega]$. By Lemma 8 , we have $f(\omega)$ is a convex quadratic function. Combining this with (51), using Lemma 19 with $d=0$, this yields the following result

$$
\operatorname{dist}\left(\omega, S_{1}\right) \leq c_{6}\left\|[f(\omega)]_{+}\right\|, \quad \forall \omega \in R^{2 m} \text {, }
$$

where $c_{6}$ is a positive constant.

Obviously, $S_{1}$ is a closed convex set. Thus, for any $\omega \in R^{2 m}$, there exists a vector $\bar{\omega} \in S_{1}$ such that

$$
\|\omega-\bar{\omega}\|=\operatorname{dist}\left(\omega, S_{1}\right)
$$

For convenience, we also let

$$
\begin{array}{r}
\Psi(\omega)=(-A(I, 0) \omega,-B(I, 0) \omega,-U(0, I) \omega \\
-V(0, I) \omega, B(I, 0) \omega, V(0, I) \omega)_{+} \cdot
\end{array}
$$

From (50), we have $\Omega^{*}=\Omega \bigcap S_{1}$, where $\Omega$ is defined in (24), so for any $\omega \in S_{1}$, combining Lemma 10 , one has

$$
\begin{aligned}
& \operatorname{dist}\left(\omega, \Omega^{*}\right) \leq \mathcal{c}_{7}[\left\|(-A(I, 0) \omega)_{+}\right\|+\left\|(-U(0, I) \omega)_{+}\right\| \\
&+\|B(I, 0) \omega\|+\|V(0, I) \omega\|] \\
&=c_{7}[\left\|(-A(I, 0) \omega)_{+}\right\|+\left\|(-U(0, I) \omega)_{+}\right\| \\
&+\left\|(B(I, 0) \omega)_{+}\right\|+\left\|(-B(I, 0) \omega)_{+}\right\|\left.+\left\|(V(0, I) \omega)_{+}\right\|+\left\|(-V(0, I) \omega)_{+}\right\|\right] \\
& \leq \mathcal{c}_{7}\left\{\left\|(-A(I, 0) \omega)_{+}\right\|_{1}+\left\|(-U(0, I) \omega)_{+}\right\|_{1}\right. \\
&+\left\|(B(I, 0) \omega)_{+}\right\|_{1}+\left\|(-B(I, 0) \omega)_{+}\right\|_{1} \\
&\left.+\left\|(V(0, I) \omega)_{+}\right\|_{1}+\left\|-(V(0, I) \omega)_{+}\right\|_{1}\right\} \\
&=c_{7}\|\Psi(\omega)\|_{1} \\
& \leq c_{7} \sqrt{2 s+2 t+2 m}\|\Psi(\omega)\|,
\end{aligned}
$$

where $c_{7}$ is a positive constant, and the second and third inequalities follow from the fact that $\|x\| \leq\|x\|_{1} \leq \sqrt{n}\|x\|$, for all $x \in R^{n}$.

Furthermore,

$$
\begin{aligned}
& \|\Psi(\omega)-\Psi(\bar{\omega})\| \\
& =\|(-A(I, 0) \omega,-B(I, 0) \omega,-U(0, I) \omega, \\
& -V(0, I) \omega, B(I, 0) \omega, V(0, I) \omega)_{+} \\
& -(-A(I, 0) \bar{\omega},-B(I, 0) \bar{\omega},-U(0, I) \bar{\omega}, \\
& -V(0, I) \bar{\omega}, B(I, 0) \bar{\omega}, V(0, I) \bar{\omega})_{+} \| \\
& =\| P_{R_{+}^{2 s+2 t+2 m}}\{(-A(I, 0) \omega,-B(I, 0) \omega, \\
& -U(0, I) \omega,-V(0, I) \omega, \\
& B(I, 0) \omega, V(0, I) \omega)\} \\
& -P_{R_{+}^{2 s+2 t+2 m}}\{(-A(I, 0) \bar{\omega},-B(I, 0) \bar{\omega}, \\
& -U(0, I) \bar{\omega},-V(0, I) \bar{\omega}, \\
& B(I, 0) \bar{\omega}, V(0, I) \bar{\omega})\} \| \\
& \leq \|\{(-A(I, 0) \omega,-B(I, 0) \omega,-U(0, I) \omega, \\
& -V(0, I) \omega, B(I, 0) \omega, V(0, I) \omega)\} \\
& -\{(-A(I, 0) \bar{\omega},-B(I, 0) \bar{\omega},-U(0, I) \bar{\omega}, \\
& -V(0, I) \bar{\omega}, B(I, 0) \bar{\omega}, V(0, I) \bar{\omega}\} \| \\
& \leq\|A(I, 0) \omega-A(I, 0) \bar{\omega}\| \\
& +2\|B(I, 0) \omega-B(I, 0) \bar{\omega}\| \\
& +\|U(0, I) \omega-U(0, I) \bar{\omega}\| \\
& +2\|V(0, I) \omega-V(0, I) \bar{\omega}\|
\end{aligned}
$$




$$
\begin{aligned}
\leq & (\|A(I, 0)\|+2\|B(I, 0)\|+\|U(0, I)\|+2\|V(0, I)\|) \\
& \times\|\omega-\bar{\omega}\| \\
= & (\|A(I, 0)\|+2\|B(I, 0)\|+\|U(0, I)\|+2\|V(0, I)\|) \\
& \times \operatorname{dist}\left(\omega, S_{1}\right),
\end{aligned}
$$

where the second equality follows from the fact that

$$
\min \{a, b\}=a-P_{R_{+}}(a-b), \quad \forall a, b \in R
$$

and the first inequality is by nonexpanding property of projection operator. Thus,

$$
\begin{aligned}
\|\Psi(\bar{\omega})\| \leq & \|\Psi(\omega)\| \\
& +(\|A(I, 0)\|+2\|B(I, 0)\| \\
& +\|U(0, I)\|+2\|V(0, I)\|) \operatorname{dist}\left(\omega, S_{1}\right) .
\end{aligned}
$$

Combining (56) with (59), for any $\omega \in R^{2 m}$, we have

$$
\begin{aligned}
\operatorname{dist}\left(\omega, \Omega^{*}\right) \leq & \operatorname{dist}\left(\omega, S_{1}\right)+\operatorname{dist}\left(\bar{\omega}, \Omega^{*}\right) \\
\leq & \operatorname{dist}\left(\omega, S_{1}\right)+\sigma\|\Psi(\bar{\omega})\| \leq \operatorname{dist}\left(\omega, S_{1}\right) \\
+ & \sigma(\|\Psi(\omega)\| \\
& +(\|A(I, 0)\|+2\|B(I, 0)\|+\|U(0, I)\| \\
& \left.+2\|V(0, I)\|) \operatorname{dist}\left(\omega, S_{1}\right)\right) \\
\leq & \sigma\|\Psi(\omega)\| \\
& +[\sigma(\|A(I, 0)\|+2\|B(I, 0)\| \\
& \quad+\|U(0, I)\|+2\|V(0, I)\|)+1] \\
& \times \operatorname{dist}\left(\omega, S_{1}\right) \\
\leq & \sigma\|\Psi(\omega)\| \\
& +[\sigma(\|A(I, 0)\|+2\|B(I, 0)\| \\
& +\|U(0, I)\|+2\|V(0, I)\|)+1] c_{6} \\
& \times\left\|[f(\omega)]_{+}\right\| \\
\leq & \eta\left(\|\Psi(\omega)\|+\left\|[f(\omega)]_{+}\right\|\right) \\
\leq & \eta\left(\|\Psi(\omega)\|_{1}+\left\|[f(\omega)]_{+}\right\|\right) \\
\leq & \eta\left(\left\|(-A(I, 0) \omega)_{+}\right\|_{1}+\left\|(-U(0, I) \omega)_{+}\right\|_{1}\right. \\
& \left.+\|B(I, 0) \omega\|_{1}+\|V(0, I) \omega\|_{1}+\left\|[f(\omega)]_{+}\right\|\right) \\
\leq & \eta\left(\sqrt{s}\left\|(-A(I, 0) \omega)_{+}\right\|+\sqrt{s}\left\|(-U(0, I) \omega)_{+}\right\|\right. \\
& +\sqrt{t}\|B(I, 0) \omega\|+\sqrt{m}\|V(0, I) \omega\| \\
& \left.+\left\|[f(\omega)]_{+}\right\|\right)
\end{aligned}
$$

$$
\begin{aligned}
\leq c_{8}( & \left\|(-A(I, 0) \omega)_{+}\right\|+\left\|(-U(0, I) \omega)_{+}\right\| \\
& +\|B(I, 0) \omega\| \\
& \left.+\|V(0, I) \omega\|+\left\|[f(\omega)]_{+}\right\|\right),
\end{aligned}
$$

where the second inequality follows from (56) with constant $\sigma=c_{7} \sqrt{2 s+2 t+2 m}$, the third inequality uses (59), the fifth inequality follows from (53), the sixth inequality follows from the fact that

$$
\begin{gathered}
\eta=\max \{\sigma,[\sigma(\|A(I, 0)\|+2\|B(I, 0)\|+\|U(0, I)\| \\
\left.+2\|V(0, I)\|)+1] c_{6}\right\},
\end{gathered}
$$

the seventh and ninth inequalities follow from the fact that

$$
\|x\| \leq\|x\|_{1} \leq \sqrt{n}\|x\|, \quad \forall x \in R^{n},
$$

and the last inequality follows by letting $c_{8}=\eta \max \{\sqrt{s}, \sqrt{t}$, $\sqrt{m}, 1\}$.

For any $x \in R^{n}$, letting $\omega:=(\mu, \nu)=(F(x), G(x)) \in R^{2 m}$, then there exists $\omega^{*}=\left(\mu^{*}, \nu^{*}\right)=\left(F\left(x^{*}\right), G\left(x^{*}\right)\right) \in \Omega^{*}$ such that $\operatorname{dist}\left(\omega, \Omega^{*}\right)=\left\|\omega-\omega^{*}\right\|$, and a direct computation yields that

$$
\begin{aligned}
& \operatorname{dist}^{1+\gamma}\left(x, X^{*}\right) \leq\left\|x-x^{*}\right\|^{1+\gamma} \\
& \leq \frac{1}{2 c_{1} \alpha^{1+\gamma}} \operatorname{dist}^{2}\left(\omega, \Omega^{*}\right) \\
& \leq \frac{1}{2 c_{1} \alpha^{1+\gamma}} c_{8}^{2} \\
& \times\left\{\left\|[A(I, 0) \omega]_{-}\right\|+\left\|[U(0, I) \omega]_{-}\right\|\right. \\
& \quad+\|B(I, 0) \omega\|+\|V(0, I) \omega\| \\
&= \frac{1}{2 c_{1} \alpha^{1+\gamma}} c_{8}^{2}\left\{\left\|[A \mu]_{-}\right\|+\left\|[U \nu]_{-}\right\|+\|B \mu\|\right. \\
&\left.\quad+\left\|\left\{[(I, 0) \omega]^{\top}[(0, I) \omega]\right\}_{+}\right\|\right\}^{2} \\
& \\
&\left.\quad+\left\|\left\{\mu^{\top} \nu\right\}_{+}\right\|\right\}^{2},
\end{aligned}
$$

where the deduction of the second equality uses the similar technique to that of (30), and the third inequality is by (60). By (63) and letting $\rho_{3}=\left\{\left(1 / 2 c_{1} \alpha^{1+\gamma}\right) c_{8}^{2}\right\}^{1 /(1+\gamma)}$, then the desired result follows.

Remark 21 . When $F$ is strongly monotone with respect to $G$, that is, $\gamma=1$, without the requirement of nondegenerate solution, the square root term in the error bound estimation is removed as stated in Theorem 20 . Hence, the error estimation becomes more practical than that in Theorem 4.1 in [4]. 


\section{Global Error Bound for the GLCP}

In this section, we consider the linear case of the GCP such that mappings $F$ and $G$ are both linear; that is, $F(x)=M x+p$, $G(x)=N x+q$ with $M, N \in R^{m \times n}, p, q \in R^{m}$ :

$$
\begin{array}{ll}
\min & H(x)=(M x+p)^{\top}(N x+q) \\
\text { s.t. } & x \in X,
\end{array}
$$

where

$$
X=\left\{x \in R^{n} \mid \begin{array}{c}
A(M x+p) \geq 0, U(N x+q) \geq 0 \\
B(M x+p)=0, V(N x+q)=0
\end{array}\right\} .
$$

For problem (64), combining (18) with (23) and using a similar discussion in Lemmas 8 and 9, we also have the following conclusion.

Lemma 22. Under Assumption 3(A1), $H(x)$ is a convex function.

Lemma 23. $x^{*} \in R^{n}$ is a solution of the GLCP if and only if $x^{*}$ is global optimal solution with the objective vanishing of (64).

Based on (64), using the argument similar to that of Theorem 13, we can obtain the following conclusion.

Theorem 24. Under Assumptions 3(A1) and 3(A2), and that mappings $F$ and $G$ are both linear, there exists constant $\rho_{4}>0$ such that

$$
\begin{aligned}
\operatorname{dist}\left(x, X^{*}\right) \leq \rho_{4}\{ & \left\|[A F(x)]_{-}\right\|+\|B F(x)\|+\left\|[U G(x)]_{-}\right\| \\
& +\|V G(x)\|+\left|\left[F(x)^{\top} G(x)\right]_{+}\right| \\
& \left.+\left|\left[F(x)^{\top} G(x)\right]_{+}\right|^{1 / 2}\right\}, \quad \forall x \in R^{n} .
\end{aligned}
$$

Proof. For any $x \in R^{n}$, a direct computation yields that

$$
\begin{aligned}
& \operatorname{dist}\left(x, X^{*}\right) \\
& \leq c_{9} \max \left\{\operatorname{dist}(x, X),\left|[H(x)]_{+}\right|,\left|[H(x)]_{+}\right|^{1 / 2}\right\} \\
& \leq c_{9} \max \left\{c _ { 1 0 } \left\{\left\|[A F(x)]_{-}\right\|+\|B F(x)\|\right.\right. \\
&\left.+\left\|[U G(x)]_{-}\right\|+\|V G(x)\|\right\}, \\
&\left.\left|[H(x)]_{+}\right|,\left|[H(x)]_{+}\right|^{1 / 2}\right\} \\
& \leq c_{9} \max \left\{c_{10}, 1\right\}\left\{\left\|[A F(x)]_{-}\right\|+\|B F(x)\|\right. \\
&+\left\|[U G(x)]_{-}\right\| \\
&+\|V G(x)\|+\left|\left[(F(x))^{\top} G(x)\right]_{+}\right| \\
&\left.+\left|\left[(F(x))^{\top} G(x)\right]_{+}\right|^{1 / 2}\right\},
\end{aligned}
$$

where the first inequality follows from Lemma 11 with constant $c_{9}>0$ and Lemma 23, and the second inequality uses Lemma 10 with constant $c_{10}>0$. By (67) and letting $\rho_{4}=$ $c_{9} \max \left\{c_{10}, 1\right\}$, the desired result follows.

Remark 25. Obviously, Assumption 3(A2) in Theorem 24 is weaker than Assumption (A2) in Theorem 4.1 in [4], Assumption 3(A1) coincides with Assumption (A1) in [4]. In addition, Theorem 24 is sharper than Theorem 4.1 in [4].

The following result further estimates the error bound for the GLCP.

Theorem 26. Suppose that the hypotheses of Theorem 24 hold, and the GLCP has a nondegenerate solution. Then, there exists constant $\rho_{5}>0$ such that

$$
\begin{array}{r}
\operatorname{dist}\left(x, X^{*}\right) \leq \rho_{5}\left\{\left\|[A F(x)]_{-}\right\|+\|B F(x)\|+\left\|[U G(x)]_{-}\right\|\right. \\
\left.+\|V G(x)\|+\left|\left[F(x)^{\top} G(x)\right]_{+}\right|\right\}, \\
\forall x \in R^{n} .
\end{array}
$$

Proof. From Corollary 17, we have

$$
\begin{array}{r}
X^{*}=\left\{x \in X \mid(M x+p)^{\top}\left(N x_{0}+q\right)\right. \\
\left.+(N x+q)^{\top}\left(M x_{0}+p\right) \leq 0\right\},
\end{array}
$$

where $x_{0}$ is a nondegenerate solution of GLCP, and $X$ is defined in (64). For any $x \in R^{n}$, a direct computation yields that

$$
\begin{aligned}
\operatorname{dist}\left(x, X^{*}\right) \leq c_{11}\{ & \left\|[A(M x+p)]_{-}\right\|+\left\|[U(N x+q)]_{-}\right\| \\
+ & \|B(M x+p)\|+\|V(N x+q)\| \\
+ & \|\left[(M x+p)^{\top}\left(N x_{0}+q\right)\right. \\
\left.\left.+(N x+q)^{\top}\left(M x_{0}+p\right)\right]_{+} \|\right\} & \\
\leq c_{11}\{ & \left\|[A(M x+p)]_{-}\right\|+\left\|[U(N x+q)]_{-}\right\| \\
& +\|B(M x+p)\|+\|V(N x+q)\| \\
& \left.+\left\|\left[(M x+p)^{\top}(N x+q)\right]_{+}\right\|\right\},
\end{aligned}
$$

where the first inequality follows from Lemma 10 with constant $c_{11}>0$, and the second inequality uses (36). Letting $\rho_{5}=c_{11}$, the desired result follows.

Remark 27. The condition in Theorem 26 is weaker than that in Theorem 4.2 in [4]. 
Theorem 28. Suppose that the hypotheses of Theorem 24 hold, and there exists point $\widehat{x} \in R^{n}$ such that (51) holds. Then there exists constant $\rho_{6}>0$ such that

$$
\begin{array}{r}
\operatorname{dist}\left(x, X^{*}\right) \leq \rho_{6}\left\{\left\|[A F(x)]_{-}\right\|+\|B F(x)\|+\left\|[U G(x)]_{-}\right\|\right. \\
\left.+\|V G(x)\|+\left|\left[F(x)^{\top} G(x)\right]_{+}\right|\right\}, \\
\forall x \in R^{n} .
\end{array}
$$

Proof. Let $S_{2}:=\left\{x \in R^{n} \mid H(x) \leq 0\right\}$, where $H(x)=$ $(M x+p)^{\top}(N x+q)$. By Lemma $22, H(x)$ is a convex quadratic function, and $S_{2}$ is a closed convex set. For any $x \in R^{n}$, there exists a vector $\bar{x} \in S_{2}$ such that

$$
\|x-\bar{x}\|=\operatorname{dist}\left(x, S_{2}\right) .
$$

Combining (51) and applying Lemma 19 yield the following result:

$$
\operatorname{dist}\left(x, S_{2}\right) \leq c_{12}\left\|[H(x)]_{+}\right\|, \quad \forall x \in R^{n},
$$

where $c_{12}$ is a positive constant. For convenience, we let

$$
\begin{aligned}
\varphi(x)= & (-A F(x),-B F(x),-U G(x) \\
& -V G(x), B F(x), V G(x))_{+} .
\end{aligned}
$$

From (50), we have $X^{*}=X \bigcap S_{2}$, where $X$ is defined in (64). So for any $x \in S_{2}$, combining Lemma 10 and using the similar technique to that of (56), one has

$$
\begin{gathered}
\operatorname{dist}\left(x, X^{*}\right) \leq c_{13}\left[\left\|(-A F(x))_{+}\right\|+\left\|(-U G(x))_{+}\right\|\right. \\
+\|B F(x)\|+\|V G(x)\|] \\
\leq c_{13} \sqrt{2 s+2 t+2 m}\|\varphi(x)\|,
\end{gathered}
$$

where $c_{13}$ is a positive constant.

Using the fact that

$$
\min \{a, b\}=a-P_{R_{+}}(a-b), \quad \forall a, b \in R,
$$

and using the similar technique to that of (57), one has

$$
\begin{aligned}
& \|\varphi(x)-\varphi(\bar{x})\| \\
& =\|(-A F(x),-B F(x),-U G(x),-V G(x), \\
& \quad B F(x), V G(x))_{+} \\
& -(-A F(\bar{x}),-B F(\bar{x}),-U G(\bar{x}), \\
& \quad-V G(\bar{x}), B F(\bar{x}), V G(\bar{x}))_{+} \| \\
& \leq\|A F(x)-A F(\bar{x})\|+2\|B F(x)-B F(\bar{x})\| \\
& +\|U G(x)-U G(\bar{x})\|+2\|V G(x)-V G(\bar{x})\| \\
& \leq(\|A M\|+2\|B M\|+\|U N\|+2\|V N\|)\|x-\bar{x}\| \\
& =(\|A M\|+2\|B M\|+\|U N\| \\
& +2\|V N\|) \operatorname{dist}\left(x, S_{2}\right),
\end{aligned}
$$

where the second inequality is by nonexpanding property of projection operator. Thus,

$$
\begin{aligned}
\|\varphi(\bar{x})\| \leq\|\varphi(x)\|+ & (\|A M\|+2\|B M\| \\
& +\|U N\|+2\|V N\|) \operatorname{dist}\left(x, S_{2}\right) .
\end{aligned}
$$

Combining (75) with (78), we know that for any $x \in R^{n}$, it holds that

$$
\begin{aligned}
& \operatorname{dist}\left(x, X^{*}\right) \\
& \leq \operatorname{dist}\left(x, S_{2}\right)+\operatorname{dist}\left(\bar{x}, X^{*}\right) \\
& \leq \operatorname{dist}\left(x, S_{2}\right)+\sigma_{1}\|\varphi(\bar{x})\| \\
& \leq \operatorname{dist}\left(x, S_{2}\right) \\
& +\sigma_{1}(\|\varphi(x)\|+(\|A M\|+2\|B M\| \\
& \left.+\|U N\|+2\|V N\|) \operatorname{dist}\left(x, S_{2}\right)\right) \\
& \leq \sigma_{1}\|\varphi(x)\| \\
& +\left[\sigma_{1}(\|A M\|+2\|B M\|\right. \\
& +\|U N\|+2\|V N\|)+1] \operatorname{dist}\left(x, S_{2}\right) \\
& \leq \sigma_{1}\|\varphi(x)\| \\
& +\left[\sigma_{1}(\|A M\|+2\|B M\|\right. \\
& +\|U N\|+2\|V N\|)+1] c_{12}\left\|[H(x)]_{+}\right\| \\
& \leq \eta_{1}\left(\|\varphi(x)\|+\left\|[H(x)]_{+}\right\|\right) \\
& \leq \eta_{1}\left(\|\varphi(x)\|_{1}+\left\|[H(x)]_{+}\right\|\right) \\
& \leq \eta_{1}\left(\left\|(-A F(x))_{+}\right\|_{1}+\left\|(-U G(x))_{+}\right\|_{1}\right. \\
& \left.+\|B F(x)\|_{1}+\|V G(x)\|_{1}+\left\|[H(x)]_{+}\right\|\right) \\
& \leq \eta_{1}\left(\sqrt{s}\left\|(-A F(x))_{+}\right\|+\sqrt{s}\left\|(-U G(x))_{+}\right\|\right. \\
& \left.+\sqrt{t}\|B F(x)\|+\sqrt{m}\|V G(x)\|+\left\|[H(x)]_{+}\right\|\right) \\
& \leq \rho_{6}\left(\left\|(-A F(x))_{+}\right\|+\left\|(-U G(x))_{+}\right\|+\|B F(x)\|\right. \\
& \left.+\|V G(x)\|+\left\|[H(x)]_{+}\right\|\right),
\end{aligned}
$$

where the second inequalities follows from (75) with constant $\sigma_{1}=c_{13} \sqrt{2 s+2 t+2 m}$, the third inequality follows from (78), the fifth inequality follows from (73), the sixth inequality follows by letting $\eta_{1}=\max \left\{\sigma_{1},\left[\sigma_{1}(\|A M\|+2\|B M\|+\|U N\|+\right.\right.$ $\left.2\|V N\|)+1] c_{12}\right\}$, and the seventh and ninth inequality follow from the fact that

$$
\|x\| \leq\|x\|_{1} \leq \sqrt{n}\|x\|, \quad \forall x \in R^{n} .
$$

By (79) and letting $\rho_{6}=\eta_{1} \max \{\sqrt{s}, \sqrt{t}, \sqrt{m}, 1\}$, the desired result follows. 
Remark 29. In Theorem 28, without the requirement of nondegenerate solution, the square root term in the error bound estimation is removed. Hence, the error estimation becomes more practical than that in Theorem 4.1 in [4].

\section{Comparison with Existing Error Bound}

In the end of this paper, we will present an example to compare Theorem 13 and Theorem 2.5 in [5]. Furthermore, we will present two examples to show the conclusion in Theorem 13 can provide a global error bound for the GNCP, while the conclusion in Theorem 2.5 in [5] cannot do.

Example 30. When $\mathscr{K}=R_{+}^{m}$, (2) reduces to the generalized nonlinear complementarity problem of finding vector $x^{*} \epsilon$ $R^{n}$ such that

$$
F\left(x^{*}\right) \geq 0, \quad G\left(x^{*}\right) \geq 0, \quad F\left(x^{*}\right)^{\top} G\left(x^{*}\right)=0 .
$$

For (81), using Theorem 13 with $\gamma=1$, we have

$$
\operatorname{dist}\left(x, X^{*}\right) \leq \tilde{\rho} \varphi(x), \quad \forall x \in R^{n},
$$

where $\varphi(x)=:\left\|[F(x)]_{-}\right\|+\left\|[G(x)]_{-}\right\|+\left|\left[F(x)^{\top} G(x)\right]_{+}\right|+$ $\left|\left[F(x)^{\top} G(x)\right]_{+}\right|^{1 / 2}$.

Using Theorem 2.5 in [5] with $\gamma=1, v_{1}=v_{2}=1$, and $\beta=1$, we have that there exists constant $\bar{\rho}>0$ such that

$$
\operatorname{dist}\left(x, X^{*}\right) \leq \bar{\rho} r(x) \text {, }
$$

where $r(x)=:\|\min \{F(x), G(x)\}\|$. In addition,

$$
\varphi(x) \leq\{2 \sqrt{m}+\|\max \{F(x), G(x)\}\|\}\left\{r(x)+r(x)^{1 / 2}\right\} .
$$

In particular, when $\|x\| \leq c_{14}$ with constant $c_{14}>0$, then there exists positive constant $c_{15}$ such that

$$
\varphi(x) \leq c_{15}\left\{r(x)+r(x)^{1 / 2}\right\}
$$

In fact, we have

$$
\begin{aligned}
\varphi(x) \leq & \left\|(F(x))_{-}\right\|_{1}+\left\|(G(x))_{-}\right\|_{1} \\
& +\left[(F(x))^{\top} G(x)\right]_{+}+\left[(F(x))^{\top} G(x)\right]_{+}^{1 / 2} \\
\leq & 2 \sqrt{m}\|\min \{F(x), G(x)\}\| \\
& +\left[(F(x))^{\top} G(x)\right]_{+}+\left[(F(x))^{\top} G(x)\right]_{+}^{1 / 2} \\
\leq & 2 \sqrt{m}\|\min \{F(x), G(x)\}\| \\
& +\sum_{i=1}^{m}\left[(F(x))_{i}(G(x))_{i}\right]_{+} \\
& +\left\{\sum_{i=1}^{m}\left[(F(x))_{i}(G(x))_{i}\right]_{+}\right\}
\end{aligned}
$$

$$
\begin{aligned}
\leq & 2 \sqrt{m}\|\min \{F(x), G(x)\}\| \\
& +\sum_{i=1}^{m}\left|\min \{F(x), G(x)\}_{i}\right| \cdot\left|\max \{F(x), G(x)\}_{i}\right| \\
& +\left\{\sum_{i=1}^{m}\left|\min \{F(x), G(x)\}_{i}\right|\right. \\
\leq & 2 \sqrt{m}\|\min \{F(x), G(x)\}\| \\
& +\|\min \{F(x), G(x)\}\| \cdot\|\max \{F(x), G(x)\}\| \\
& +\{\|\min \{F(x), G(x)\}\| \cdot\|\max \{F(x), G(x)\}\|\}^{1 / 2} \\
= & \{2 \sqrt{m}+\|\max \{F(x), G(x)\}\|\} r(x) \\
& +\|\max \{F(x), G(x)\}\|^{1 / 2} r(x)^{1 / 2} \\
\leq & \{2 \sqrt{m}+\|\max \{F(x), G(x)\}\|\}\left\{r(x)+r(x)^{1 / 2}\right\}
\end{aligned}
$$

where the first inequality follows from the fact that $\|x\| \leq$ $\|x\|_{1}$, for all $x \in R^{m}$, the second inequality follows from the fact that $a_{-} \leq|\min \{a, b\}|$, for all $a, b \in R$, the third inequality follows from the fact that $(a+b)_{+} \leq a_{+}+b_{+}$, for all $a, b \in R$, the fourth inequality follows from the fact that $(a b)_{+} \leq|\min \{a, b\}| \cdot|\max \{a, b\}|$, for all $a, b \in R$, and the fifth inequality follows from the Cauchy-Schwarz inequality.

Example 31. For mappings $F, G: R_{+} \rightarrow R$ involved in problem (81), we set

$$
F(x)=(x+1)^{2}, \quad G(x)=\sqrt{x} .
$$

It is easy to see that the solution set $X^{*}=\{0\}$, and one has

$$
\begin{aligned}
\langle F(x)-F(y), G(x)-G(y)\rangle & =\frac{x+y+2}{\sqrt{x}+\sqrt{y}}(x-y)^{2} \\
& \geq(x-y)^{2}
\end{aligned}
$$

where the first inequality follows from the fact that $x+y+2 \geq$ $\sqrt{x}+\sqrt{y}$.

In fact, we consider the following four cases.

Case $1(x \geq 1$ and $y \geq 1)$. Then, $x \geq \sqrt{x}$ and $y \geq \sqrt{y}$, and the desired result follows.

Case $2(0 \leq x \leq 1$ and $0 \leq y \leq 1)$. Then, $\sqrt{x} \leq 1$ and $\sqrt{y} \leq 1$, and the desired result follows.

Case $3(0 \leq x \leq 1$ and $y \geq 1)$. Then, $\sqrt{x} \leq 1$ and $\sqrt{y} \leq y$, and the desired result follows.

Case $4(x \geq 1$ and $0 \leq y \leq 1)$. Then, $\sqrt{x} \leq x$ and $\sqrt{y} \leq 1$, and the desired result follows. 
For any $x(\epsilon):=\epsilon, \epsilon \geq 0$. By Theorem 13 with $\gamma=1$, we can obtain

$$
\frac{\|x(\epsilon)-0\|}{\varphi(x(\epsilon))}=\frac{\epsilon}{(\epsilon+1)^{2} \sqrt{\epsilon}+\sqrt{(\epsilon+1)^{2} \sqrt{\epsilon}}} \longrightarrow 0
$$

as $\epsilon \rightarrow+\infty$. Thus, Theorem 13 provides a global error bound for the GNCP. Using Theorem 2.5 in [5], for $x(\epsilon)$, we have

$$
\frac{\|x(\epsilon)-0\|}{r(x(\epsilon))}=\frac{\epsilon}{\left\|\min \left\{(\epsilon+1)^{2}, \sqrt{\epsilon}\right\}\right\|}=\frac{\epsilon}{\sqrt{\epsilon}}=\sqrt{\epsilon} \longrightarrow+\infty
$$

as $\epsilon \rightarrow+\infty$. Thus, Theorem 2.5 in [5] fails in providing an error bound for this GNCP.

Example 32. For mappings $F, G: R \rightarrow R$ involved in problem (81), we set

$$
F(x)=\frac{1}{3} x^{3}+x, \quad G(x)=x .
$$

It is easy to see that the solution set $X^{*}=\{0\}$. Without loss of generality, we let $x>y$, and one has

$$
F(x)-F(y) \geq(x-y)^{2},
$$

where the inequality follows from the fact that

$$
\begin{aligned}
\left(\frac{1}{3} x^{3}+x\right)-( & \left.\frac{1}{3} y^{3}+y\right)-(x-y)^{2} \\
=(x-y) & {\left[\frac{1}{3} x^{2}+\frac{1}{3} y^{2}\right.} \\
& \left.+\frac{1}{3} x y+1+(x-y)\right] \geq 0 .
\end{aligned}
$$

In fact, we consider the following four cases.

Case $1(x>y \geq 0)$. Then, $(1 / 3) x^{2}+(1 / 3) y^{2}+(1 / 3) x y+1+$ $(x-y) \geq 0$, and the desired result follows.

Case $2(0 \geq x>y)$. Then, $(1 / 3) x^{2}+(1 / 3) y^{2}+(1 / 3) x y+1+$ $(x-y) \geq 0$, and the desired result follows.

Case $3(x \geq 0, y<0$ and $x+y \geq 0)$. Then,

$$
\begin{aligned}
\frac{1}{3} x^{2}+ & \frac{1}{3} y^{2}+\frac{1}{3} x y+1+(x-y) \\
& =\frac{1}{3} x(x+y)+\frac{1}{3} y^{2}+1+(x-y) \geq 0,
\end{aligned}
$$

and the desired result follows.

Case $4(x \geq 0, y<0$, and $x+y \leq 0)$. Then,

$$
\begin{aligned}
\frac{1}{3} x^{2}+ & \frac{1}{3} y^{2}+\frac{1}{3} x y+1+(x-y) \\
& =\frac{1}{3} x^{2}+\frac{1}{3} y(x+y)+1+(x-y) \geq 0,
\end{aligned}
$$

and the desired result follows.
Thus, we obtain

$$
\langle F(x)-F(y), G(x)-G(y)\rangle \geq(x-y)^{3} .
$$

For any $x(\epsilon):=\epsilon, \epsilon \geq 0$. By Theorem 13 with $\gamma=2$, we can obtain

$$
\frac{\|x(\epsilon)-0\|}{\varphi(x(\epsilon))}=\frac{\epsilon}{\left[\left((1 / 3) \epsilon^{3}+\epsilon\right) \epsilon+\sqrt{\left((1 / 3) \epsilon^{3}+\epsilon\right) \epsilon}\right]^{2 / 3}} \longrightarrow 0
$$

as $\epsilon \rightarrow+\infty$. Thus, Theorem 13 provides a global error bound for the GNCP.

On the other hand, using Theorem 2.5 in [5], for $x(\epsilon)$, we have

$$
\frac{\|x(\epsilon)-0\|}{r(x(\epsilon))^{\delta}}=\frac{\epsilon}{\left\|\min \left\{(1 / 3) \epsilon^{3}+\epsilon, \epsilon\right\}\right\|^{\delta}}=\frac{\epsilon}{\epsilon^{\delta}} \longrightarrow+\infty
$$

as $\epsilon \rightarrow+\infty$, where $\delta$ is a constant with $1 / 3<\delta \leq 1 / 2$. Thus, Theorem 2.5 in [5] fails in providing an error bound for this GNCP.

\section{Conclusion}

In this paper, we established some global error bounds on the generalized nonlinear complementarity problems over a polyhedral cone, which improves the result obtained for variational inequalities and the GNCP $[4,5,9-11]$ by weakening the assumptions. Surely, under milder conditions, we may establish global error bounds for GNCP and use the error bounds estimation to establish quick convergence rate of the methods for the GNCP. This is a topic for future research.

\section{Acknowledgments}

The authors wish to give their sincere thanks to the associated editor and two anonymous referees for their valuable suggestions and helpful comments which improve the presentation of the paper. This work was supported by the Natural Science Foundation of China (nos. 11171180 and 11101303), the Specialized Research Fund for the Doctoral Program of Chinese Higher Education (20113705110002), the Shandong Provincial Natural Science Foundation (ZR2010AL005), the Shandong Province Science and Technology Development Projects (2013GGA13034), and the Applied Mathematics Enhancement Program of Linyi University.

\section{References}

[1] M. A. Noor, "General variational inequalities," Applied Mathematics Letters, vol. 1, no. 2, pp. 119-122, 1988.

[2] Y. J. Wang, F. M. Ma, and J. Z. Zhang, "A nonsmooth L-M method for solving the generalized nonlinear complementarity problem over a polyhedral cone," Applied Mathematics and Optimization, vol. 52, no. 1, pp. 73-92, 2005.

[3] X. Z. Zhang, F. M. Ma, and Y. J. Wang, "A Newton-type algorithm for generalized linear complementarity problem over a polyhedral cone," Applied Mathematics and Computation, vol. 169, no. 1, pp. 388-401, 2005. 
[4] H. C. Sun, Y. J. Wang, and L. Q. Qi, "Global error bound for the generalized linear complementarity problem over a polyhedral cone," Journal of Optimization Theory and Applications, vol. 142, no. 2, pp. 417-429, 2009.

[5] H. C. Sun and Y. J. Wang, "Global error bound estimation for the generalized nonlinear complementarity problem over a closed convex cone," Journal of Applied Mathematics, vol. 2012, Article ID 245458, 11 pages, 2012.

[6] R. Andreani, A. Friedlander, and S. A. Santos, "On the resolution of the generalized nonlinear complementarity problem," SIAM Journal on Optimization, vol. 12, no. 2, pp. 303-321, 2002.

[7] F. Facchinei and J. S. Pang, Finite-Dimensional Variational Inequality and Complementarity Problems, Springer, New York, NY, USA, 2003.

[8] L. Walras, Elements of Pure Economics, Allen and Unwin, London, UK, 1954.

[9] M. V. Solodov, "Convergence rate analysis of iteractive algorithms for solving variational inquality problems," Mathematical Programming, vol. 96, no. 3, pp. 513-528, 2003.

[10] J.-S. Pang, "A posteriori error bounds for the linearly-constrained variational inequality problem," Mathematics of Operations Research, vol. 12, no. 3, pp. 474-484, 1987.

[11] N. H. Xiu and J. Z. Zhang, "Global projection-type error bounds for general variational inequalities," Journal of Optimization Theory and Applications, vol. 112, no. 1, pp. 213-228, 2002.

[12] R. A. Horn and C. R. Johnson, Topics in Matrix Analysis, Cambridge University Press, Cambridge, UK, 1991.

[13] A. J. Hoffman, "On approximate solutions of systems of linear inequalities," Journal of Research of the National Bureau of Standards, vol. 49, pp. 263-265, 1952.

[14] T. Wang and J.-S. Pang, "Global error bounds for convex quadratic inequality systems," Optimization, vol. 31, no. 1, pp. $1-12,1994$.

[15] M. A. Noor, "Merit functions for general variational inequalities," Journal of Mathematical Analysis and Applications, vol. 316, no. 2, pp. 736-752, 2006. 


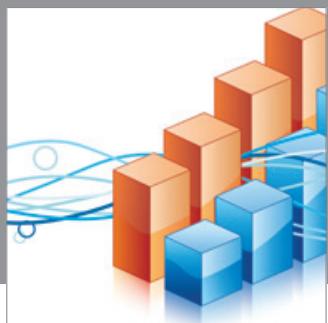

Advances in

Operations Research

mansans

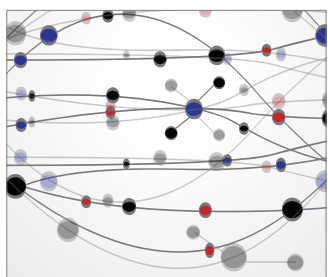

The Scientific World Journal
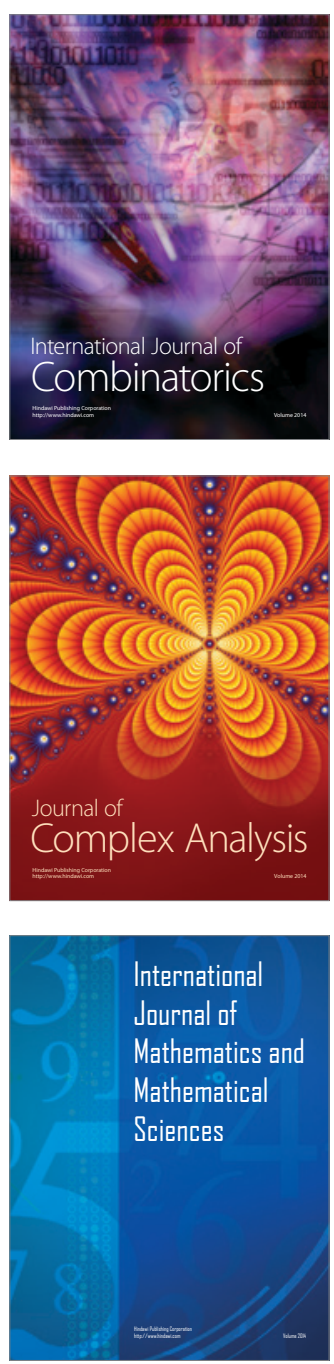
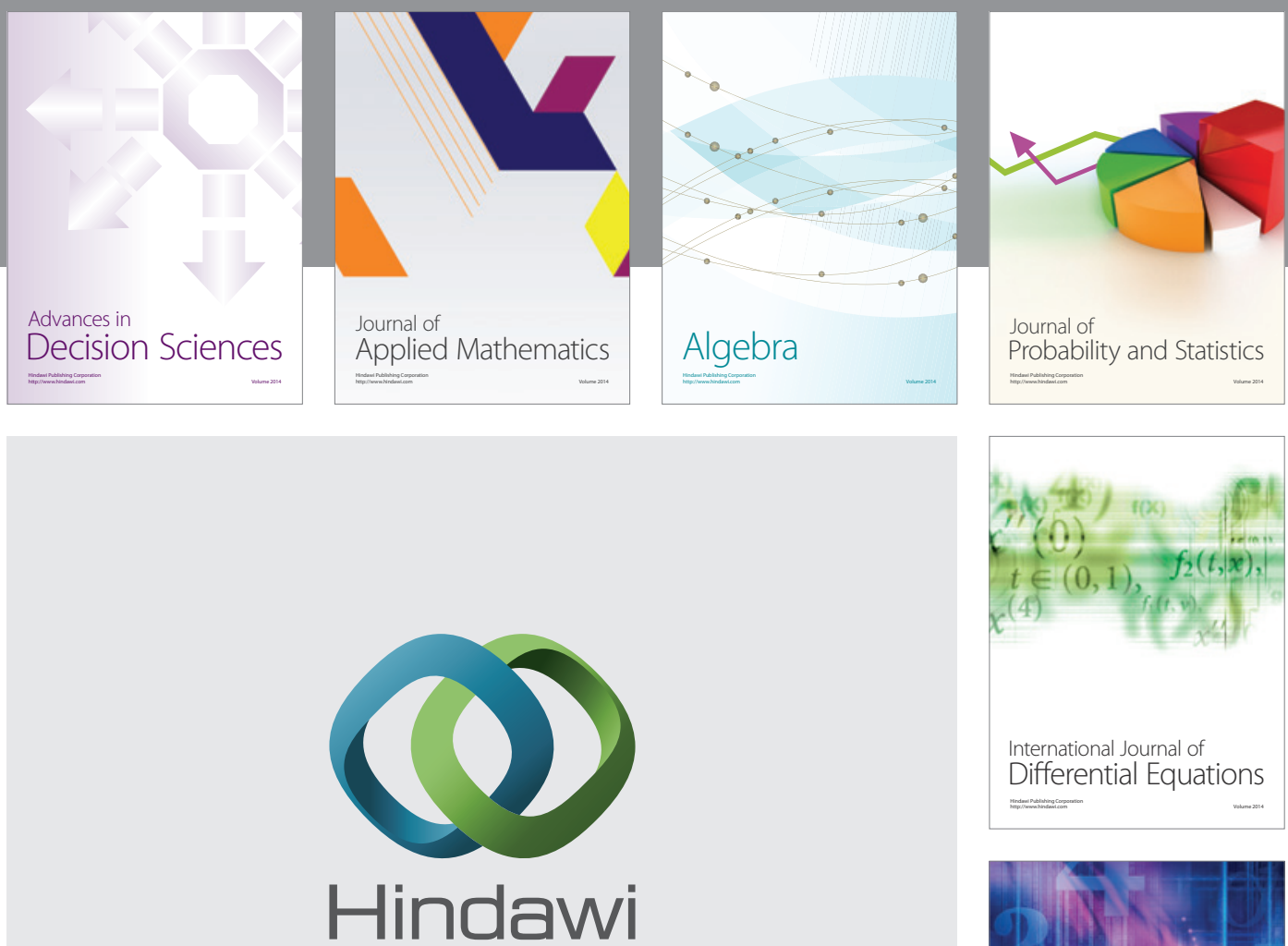

Submit your manuscripts at http://www.hindawi.com
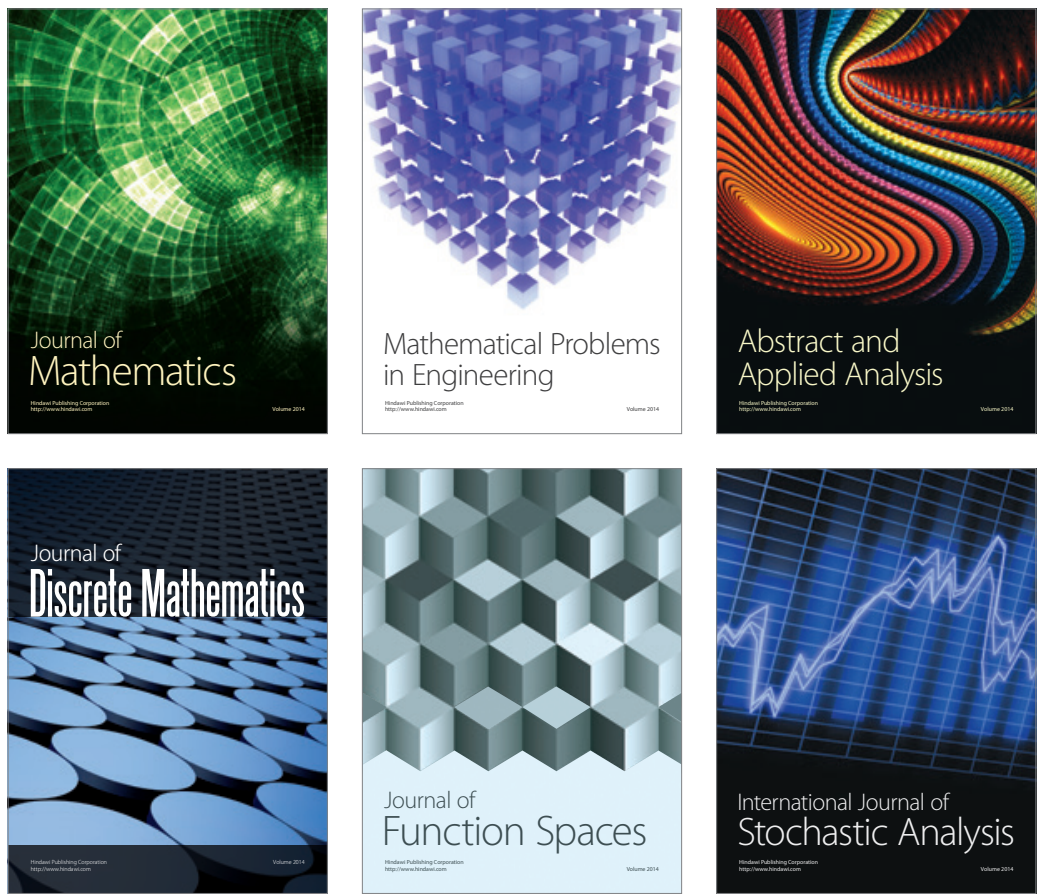

Journal of

Function Spaces

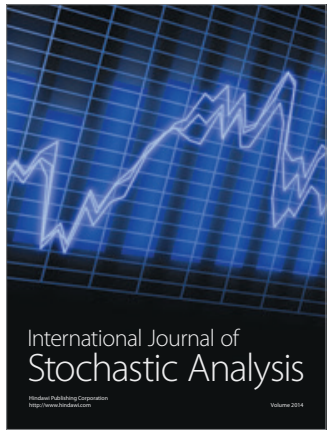

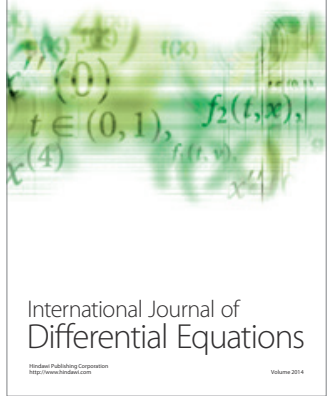
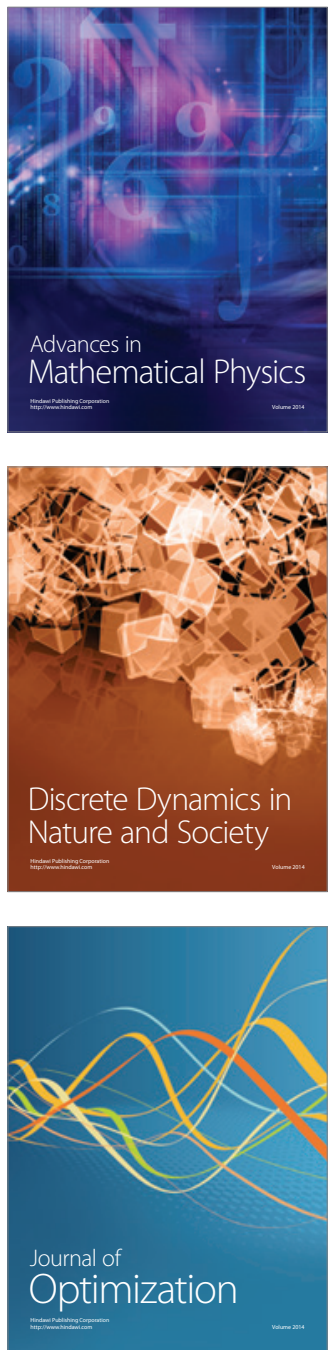